\title{
Urgences
}

\section{À mots ouverts}

\section{Élisabeth Haghebaert}

Numéro 23, avril 1989

Lisière du livre

URI : https://id.erudit.org/iderudit/025514ar

DOI : https://doi.org/10.7202/025514ar

Aller au sommaire du numéro

Éditeur(s)

Urgences

ISSN

0226-9554 (imprimé)

1927-3924 (numérique)

Découvrir la revue

Citer cet article

Haghebaert, É. (1989). À mots ouverts. Urgences, (23), 36-44.

https://doi.org/10.7202/025514ar

Ce document est protégé par la loi sur le droit d'auteur. L'utilisation des services d'Érudit (y compris la reproduction) est assujettie à sa politique d'utilisation que vous pouvez consulter en ligne.

https://apropos.erudit.org/fr/usagers/politique-dutilisation/
Cet article est diffusé et préservé par Érudit.

Érudit est un consortium interuniversitaire sans but lucratif composé de l’Université de Montréal, l'Université Laval et l'Université du Québec à Montréal. Il a pour mission la promotion et la valorisation de la recherche. https://www.erudit.org/fr/ 


\section{ÉLISABETH HAGHEBAERT \\ À mots ouverts}

Du paratexte à la paraphrase, «couverture» pour une ouverture

Étant donnés:

1 l'objet: le déjà-là, le déjà-fait, incontournable forcément:

“(al)ready-made», volume et surface, palpable et tangible, le

livre livré en lecture au lecteur, ready to (be) read;

$2^{\circ}$ le sujet: livre de (André Gervais, auteur et critique), d'une part,

livre sur (Marcel Duchamp, «artiste et anartiste»), d'autre part;

Le passage de l'un à l'autre: l'objet fait sujet, et vice versa, force est de sérier l'approche. Face à l'envergure de l'ensemble et compte tenu du type du livre - une analyse détaillée du littéraire et du pictural chez Marcel Duchamp et une somme d'effets de langue -, on se limitera, sur "simple décision de méthode»", aux extérieurs, aux marches du texte: le paratexte, au sens où l'accessoire inclut l'accès, comme voie d'approche donc.

Là encore, il faut préciser, délimiter, éliminer. Entre dédicace quintipare $^{3}$ - trajet du professionnel à l'affectif —, épigraphes d'ouverture (p. 1) et de fermeture (p. 411) éclairées et éclairantes, françaises et anglaise, qui traitent, entre autres ${ }^{4}$, de scansion, de calembour et d'acrostiche, table des matières triptyque et cryptique et - dans la zone trouble d'indécision entre texte et paratexte - protubérances et modulations de l'appareil de notes, se résoudre à reconnaître que rendre compte d'un tel livre est une entreprise aussi décourageante que passionnante: une invite à pénétrer les arcanes de la recherche.

La teneur informative des renseignements produits par le rabat à propos de l'illustration de la jaquette ${ }^{5}$ (titre, date, nature et source des documents) fournit un échantillon que confirment index et bibliographie: le livre de Gervais, en dépit de la désinvolture ou de la modestie du sous-titre («Apropos of...»), constitue un solide ouvrage de référence sur l'oeuvre de Duchamp ${ }^{6}$.

L'analyse minutieuse ef érudite que mène l'auteur (p. 119127 , particulièrement) à propos du seul objet Pollyperruque ${ }^{7}$ qui apparaît en illustration rend évidemment présomptueuse la tenta- 
tive néophyte de la lectrice: rien qui ne soit sans doute «déjà dit», rien qu'un potentiel re-dit. Suspendre pour autant l'investigation reviendrait toutefois à nier la démonstration même de la valeur didactique de la répétition, dont l'objet intitulé Pollyperruque pourrait, semble-t-il, être l'emblème. L'omission, l'oubli et toutes formes de lacunes de cette lecture de surface ne font que souligner l'intérêt du retour à l'origine et renvoient à la lecture du livre bien entendu.

Au seuil d'une démarche initiatique donc, commencer par commenter la pellicule externe, s'en tenir à l'apparence, ce qui entoure le livre et le revêt, au sens propre, de son libellé inducteur: la jaquette, ou la liseuse. Autrement dit l'occasion, pour toutes les connotations, de l'apparat à l'intime ${ }^{8}$, qui se rattachent à la métaphore vestimentaire, de devenir autant d' «effets» qui sans quoi n'auraient pas de sens. Voilà comment la nature même du support matériel permet d'établir un lien, capilliforme dirai-je, - si «tiré par les cheveux $"{ }^{9}$ soit-il justement — , entre le titre du livre - «la raie», ses "effets" - et le titre de l'oeuvre représentée: Pollyperruque. Ce rapport prend une dimension toute duchampienne quand l'intertexte restreint — l'oeuvre de Duchamp même —, révèle l'existence d'un projet de jaquette de livre, représentant précisément une jaquette, au sens figuratif du terme, c'est-à-dire un habit queue-de-pie ${ }^{10}$. Proposé par Duchamp, ce projet fut refusé comme "bad joke» - taxé de mauvais goût - par l'éditeur A.A. Knopf ${ }^{11}$. Le présent choix de Pollyperruque lui rend en quelque sorte indirectement justice - via l'infratexte, autrement dit à la dérobée - en affichant, sinon des pies ${ }^{12}$ les queues, du moins toutes sortes d'oiseaux.

Englobant une oeuvre étonnante de part et d'autre, la jaquette obéit aux indications génériques répertoriées par Gérard Genette: séduire le public potentiel tout en identifiant et désignant le contenu. Lieu de manipulation par définition, elle y parvient, à quelque réserve près, par les moyens traditionnels: le titre, La raie alitée d'effets, icône de la lecture-écriture d'André Gervais, appelant le sous-titre aussi énigmatique qu'explicatif Apropos of Marcel Duchamp, et l'illustration picturale, Pollyperruque, empruntée à l'oeuvre de Marcel Duchamp ${ }^{13}$. C'est par cette double voie que se tente l'accès à un imaginaire rigoureusement surmultiplié et contrôlé ${ }^{14}$, ouvert à tous les possibles du sens, voire du non-sens.

Affichée la couleur, observons donc, avant de revenir à la polychromie et à la textualité proprement dite, l'enveloppe de l'objet, c'est-à-dire, doublant la couverture et anticipant la lecture, 
préambule à la compréhension, le lieu de préhension. Élément indépendant, amovible et dépliable, hommage à l'éphémère, ce qui s'ôte aux yeux dès l'ouverture, la jaquette masque ou laisse à nu, le gris-beige austère - institutionnel - à peine souligné de turquoise, de la couverture. Rarement métaphore semble plus appropriée, donc, que cette jaquette qui revêt de sens, autant qu'elle le révèle, le corps et le caractère d'un ouvrage complexe. Doublure et pelure ${ }^{15}$, elle appelle, pour donner le ton et l'allure, le feuilletage qui peut-être fera détecter le leurre, ou the lure, de l'«emballage» qu'elle constitue. La jaquette est aussi, ne l'oublions pas, le revêtement qui donne l'aspect de l'émail à une couronne, c'est-à-dire son "poli». Ainsi, dans le contexte duchampien, évoque-t-elle par homonymie, Pollyperruque bien sûr, mais également, par analogie avec son propre fini glacé, cet autre readymade intitulé justement Apolinère Enameled ${ }^{16}$ (1916-1917). Bref, de l'ordre de l'installation, l'objet jaquette requiert ici d'emblée une attention qui convoque irrévocablement la participation du lecteur potentiel.

\section{De quelques à-côtés}

En premier lieu, c'est-à-dire centré tout en haut, se trouve le nom de l'auteur. La connotation géobiographique qui relie André Gervais à son sujet mérite une halte. Une lointaine prédestination disons toponomastique, par le détour hors contexte d'un PréSaint-Gervais ${ }^{17}$, renvoie en effet l'auteur (Gervais) et le sujet (Duchamp) à leurs origines linguistiques naturelles communes, la France.

Ce rapprochement anecdotique et fortuit, de l'ordre de l'«ajustage de coïncidence", selon la formule duchampienne, a pour intérêt de mettre en évidence tel autre, qui est leur respective adhérence, adoptive ou sélective, au continent nord-américain. Ceci expliquant cela, cette double appartenance justifie les opérations translinguistiques que l'un et l'autre font subir aux deux langues dont ils disposent, ainsi par exemple, l'à-propos du sous-titre Apropos of Marcel Duchamp. Cet «à propos», que l'on trouve employé tel que par Duchamp dans À propos de jeune soeur (1911) ou sous sa forme anglaise (en un seul mot) comme ici, dans Apropos of "Readymades" (1961) ou Apropos of Myself (1964), sert à unifier les trois volets du livre qui, entre les épigraphes d'ouverture et de fermeture et en amont des notes, bibliographies et index, traitent, le premier du dispositif du nom, le deuxième du ready-made, le troisième des aphorismes. 
Au même chapitre des effets conjugués du hasard et de la géographie, ajoutons enfin, pour le $"$ Witz ${ }^{18}$ caché des jeux d'esprit, que Pollyperruque appartient à un collectionneur de BadenBaden, nom de ville qui affiche à son insu, sous le répétitif, le signe de binarité obsédante caractéristique de l'oeuvre en question.

\section{Haut lieu d'intrigue, le titre}

Énoncer le titre, haut lieu d'intrigue: La raie alitée d'effets. Cet acte de baptême insolite témoignant d'un parti pris d'analphabétisme ou de brouillage délibéré incite à la circonspection. Le «message» n'est peut-être pas celui que l'on donne pour tel. Sémantiquement surchargé, dans «la réalité des faits», le propos est trop clair pour être sans équivoque: le calembour désigne l'ambiguitté érigée en règle et élimine toute immotivation initiale. Tout est de l'ordre d'un calcul où rien n'est gratuit: le hasard même semble concerté, à commencer par la citation éditoriale, de Brecht, en adéquation parfaite avec la démarche de Gervais qui, par les brèches qu'il inflige lui-même au sens établi, met à jour les "raisons opposées" aux "bonnes raisons" ${ }^{19}$.

Si délibérément l'on élude l'aspect paillard - éroticoagriculturel par trop évident - de la raie, l'opération de racolage titulaire demande à être considérée comme telle à un second niveau. C'est-à-dire bel et bien à titre d'appeau, "La peau de la raie» de Roussel bien sûr, comme ne manque pas de le noter Gervais ${ }^{20}$. Elle invite alors à pénétrer les rets du texte et à y inventorier les multiples charades à tiroirs systématiquement analysées et explorées. Parmi celles-ci, un grand nombre de blagues de carabins ${ }^{21}$ et de gauloiseries ramènent, il faut le dire, au propos initial, mais non sans le revêtir, bien sûr, du plus grand sérieux.

De ce titre, disons, par révérence pour le joueur d'échecs Marcel Duchamp, qu'il implique de jouer la diagonale et le jeu phonique (à moins d'oser littéoral) où l'art s'engraisse aux effets graphiques pour devenir «lard» (et toutes variables dérivables). Car ici tout incline à suivre littéralement la pente du bouquineur, à pratiquer une lecture oblique, spectaculairement spéculaire et vice versa, «en effets». Ces effets visuels, autant que textuels, annoncent le produit: un texte déroutant, à l'aune de l'oeuvre de Duchamp. «Justifié» à droite, le dispositif titulaire chapeaute d'un triangle rectangle la reproduction de Pollyperruque, ainsi: 


\section{La \\ raie \\ alitée \\ d'effets \\ Apropos of Marcel Duchamp}

Blanc sur noir, à l'instar de l'alphabet des astres mallarméen, ou, moins cosmique, à l'instar de tel tableau noir qui rappelle la vocation didactique de l'ouvrage, le sens de la distorsion se manifeste ici de la même façon qu'il apparaissait dans le numéro 103 de La nouvelle barre du jour ${ }^{22}$ où parut sous forme d'article le chapitre intitulé «de l'angrais Duchamp de l'infratexte».

La disposition en calligramme minimal évoque par ailleurs l'existence parallèle d'Apolinère Enameled. Dans les mots alignés sur quelque marge imaginaire se rétablit la réalité défaite, mise à mal dans la dérive et les effets du langage. Camouflet dans les lignes ennemies du «bon" sens univoque, ce titre affiche impudiquement les camouflages de l'inconscient. L'a-réalité des fées met en scène l'effet théâtral de la polyvocité confrontée à telle raie bien réelle, préalablement ici écartée pour son sens trop (peu) équivoque. Preuve qu'à travers les errances du et des sens, telle raie prise pour cible désigne aussi l'arrêt où se manifeste le seuil de tolérance du lecteur.

À la manière du ready-made dont c'est précisément l'essence de répéter le réel, elle re-dit en tous sens, calembour compris: l'art est partout. Comme l'indique la voie éditauctoriale ${ }^{23}$ en quatrième de jaquette: le texte s'énonce comme «mise à nu de passages et de rymes (de langues, de textes, de lieux, d'époques, de sexes)". Elle le dit avec le sérieux de la farce que l'on attache au poisson d'avril. La raie sort du rang, du sillon où s'englue Eros, pour épuiser les divers possibles du champ sémantique et rétablir le lien et les enchaînements avec Pollyperruque, le ready-made ${ }^{24}$ représenté.

\section{Pollyperruque, pero qué?}

Polychrome, cette illustration occupe approximativement les deux tiers de l'espace de la première de jaquette. II s'agit en fait d'un «semi-ready-made ${ }^{25}$ » comme l'indique l'information technique mentionnée au rabat c'est-à-dire, selon Duchamp, un «assemblage, collage, d'objets tels quels ou aidés». Destinée à satisfaire la curiosité lectorale, celle-ci procure soulagement instantané, ou 
«relief», à l'anglaise, pour objet en trois dimensions. Précisons que, d'après l'index des oeuvres picturales citées dans le texte auquel il sert d'image de marque en quelque sorte, ce "semiready-made» sera mentionné à onze reprises ${ }^{26}$, incluant notes et tableaux explicatifs.

Tout comme certaines oeuvres abstraites déconcertent par la difficulté de trouver une prise par où les aborder, Pollyperruque surprend au contraire par son apparente facilité d'accès, en un mot, par son foisonnement.

Hautement figuratif, l'objet ici mis à plat peut se définir comme un collage ou un montage dont la principale caractéristique serait l'étagement des plans, et leur possible mobilité, grâce à un jeu de tirette et de charnières, ce dont rendent compte les schémas et les commentaires de son propriétaire, Dieter Keller ${ }^{27}$. Disons pour l e situer qu'il a été originellement adressé, comme le révèlent les notes, par Marcel Duchamp à Arne Ekstrom dans le cadre d'une exposition qui eut lieu à New York en 1967-1968 et dont le titre initial, «Polyimagists», devint «Polly-imagists»"

Trouée, ou «brèche» donc dans le noir total — de l'encre d'imprimerie -, Pollyperruque est présenté par Gervais comme «carte-souvenir espagnole ${ }^{29}$ avec la formule connue: Muchos Recuerdos de (Beaucoup de Souvenirs de la part de) et une étonnante signature: POLLYPERRUQUE». (p. 119)

Fragment subjectif d'un imaginaire autre, Pollyperruque apparaît sous l'espèce du composite et se signale au regardeur à la manière d'un chromo: par l'assemblage de ses couleurs criardes. Carte postale oui, mais ceci n'est qu'une partie du tout, se détachant, en relief, sur un fond constitué par une planche ornithologique ${ }^{30}$ - collée sur l'illustration originale - qui représente, entre autres, «in vivo", perroquets et perruches, tous membres - sans doute n'est-ce pas un hasard - de la famille des psittacidés ${ }^{31}$. D'autant plus percutant apparaît alors le rapprochement effectué par l'auteur entre cet objet et l'intertexte apollinarien: «Les plaques les avis à la façon des perroquets crillaient ${ }^{32}$. Ne serait-ce que motivé par les caractéristiques propres à ces oiseaux-là, le sens de la famille exhibé ici provoque bien sûr celui des familles de mots: ces oiseaux (rares) sont de beaux parleurs, "à langue épaisse et très mobile» précise le dictionnaire. Excellents imitateurs, il y a dans le fait de les représenter un effet miroir qui se répercute sur l'ensemble, comme si le fond, pour de bon, générait la forme. Dédoublement, redoublement, répétition et superposition consti- 
tuent ici l'essence de l'objet, au point de n'en faire qu'un «semiready-made». Produit semi fini d'une (dé)multiplication infinie.

À la manière d'une carte postale, particulièrement du type de celle-ci, tout fait signe, en effet. II faut dire que cette carte est un modèle théorique du genre kitsch et «sentimental»: couple d'oiseaux - de ces hirondelles ressemblant aux perruches dites «inséparables» ${ }^{33}$ - découpés et perchés sur l'anse d'un panier tressé débordant de violettes roses au dessous duquel s'étale le message linotypé, «Muchos Rucuerdos de», suivi de la signature éponyme. L'étonnant de celle-ci est qu'elle apparaît, majuscule et manuscrite, sur une sorte d'étiquette blanche surajoutée à on ne sait quoi, laissant place à l'énigme sous le gommage.

Tout concourt, on le voit, à mettre l'accent sur le surfait, le factice et la falsification. Mensonge criant des violettes roses ${ }^{34}$ par exemple, masqué par cette étrange signature qui a tout l'aspect d'un pseudonyme. Qu'indiquent ces fleurs et ces oiseaux de papier dans ce panier en trompe-l'oeil? (faut-il le nier ou pas?). La répétition du même en tous sens: montage pour coup monté.

On ne fera que jouer le même jeu en insistant sur le polyglottisme de l'ensemble qui, porté à l'indice trois par un détour à l'anglaise, montre le rapport de complémentarité intrinsèque qui existe entre «poll» (= tondre) "y» (= et, à l'espagnole) "perruque» (= imitation de chevelure). Renversée en «recuer-», voilà la perruque dédoublée dans le factice du souvenir: ce qui tient à un fil ou, parfois, à un cheveu. Coup double donc, qui s'entend dans le «deux» (le «dos» espagnol) inclus dans «recuer-dos».

Gervais ayant montré le lien qui unit, entre autres, "Polly» et perroquet par le biais du diminutif anglais généralement attribué à ces oiseaux, un dernier coup (de grâce) nous ramènera au point de départ. II s'agit d'un coup double encore puisque dans le «perroquet» s'entend un coup de chien ${ }^{35}$. «Perro» désigne en effet le chien, en espagnol; le «roquet» bien sûr. Ce petit chien qui aboie pour un rien s'apparente ainsi à la perruche dont la loquacité est attestée par l'expression familière «bavard comme une perruche» ou "bavard comme une pie". Mais ce n'est pas tout. Car avec un seul « $R{ }^{36}$, «pero» signifie encore «mais», mais «quoi?», c'est-àdire: "pero qué?»: so what?... Ou l'art mis en question par ces perroquets porteurs de leur propre interrogation. Une chose est certaine - avant de tourner casaque - «roquer » relève aussi du vocabulaire technique du jeu d'échecs, ce qui nous ramène à la diagonale et aux effets initiaux. Effets de superposition de sens qui 

sous couvert de.

1. D'après le calembour de Duchamp: «anartist", agglutination de "an + artist»; expression traduite et reprise par André Gervais en quatrième de jaquette.

2. A la manière de Gérard Genette, Seuils, Paris, Seuil, coll. «Poétique», 1987, p. 315.

3. Énumérons, par ordre alphabétique: Joseph Bonenfant, directeur de la thèse à l'origine de ce livre, et «vieil ami»; Thierry de Duve, autre docteur ès «duchampienneries», rencontré à Cerisy; Michel Gay, poète québécois, qui a «ouvert» un numéro de la $\mathbf{N B J}$ (le numéro 103, théorique, du mois de mai 1981, intitulé "L'infratexte») à des textes de Diderot, Roussel et... Duchamp [Le numéro 82, d'octobre 1979, notons-le également, avait déjà publié le texte intitulé «Du raide y m'aide»); Gilles Hénault, poète québécois et premier directeur du Musée d'art contemporain (Montréal), qui a bien conru Michel Sanouillet, l'éditeur des écrits de Marcel Duchamp; Nicole Hémond, enfin, qui, depuis 1973, connaît forcément tout de Duchamp.

4. Il faudrait bien sûr aussi parler d'érotisme, de dédoublement, etc.

5. On ne peut omettre de mentionner, dans le présent contexte paratextuel, que le rabat de la page 1 de cette jaquette confond jaquette et couverture puisqu'il y est question de l'illustration «en couverture». Notons que la couverture proprement dite, comme toutes celles de cette collection, se distingue par la neutralité de sa présentation.

6. À propos d'André Gervais, en revanche, hormis le nom et la dédicace, on remarque l'absence, sur l'autre rabat de la jaquette par exemple, de note biographique et de toute trace auctoriale.

7. Précisons, d'après les renseignements fournis par l'auteur, que ce choix a été conjoncturel. En effet, Première lumière (1959), la première illustration sélectionnée, qui - hors contexte politique québécois - mettait en scène le mot "NON» et avait été conçue pour accompagner un poème de Pierre-André Benoît, n'a pu être disponible.

8. De la jaquette de cérémonie à la liseuse que l'on met pour lire au lit, ou comment faire de l'effet avec quelques hardes pour effets.

9. Dirait Ricardou.

10. Mentionnons aussi, toujours dans la métaphore vestimentaire, le ready-made intitulé Gilet (1957-1961).

11. D'après le petit catalogue de l'exposition rétrospective qui eut lieu au Musée d'art de Philadelphie du 22.9 au 11.11. 1973 et au Musée d'Art moderne de New York du 3.121973 au 10.2 1974, une maquette, représentant une jaquette de cérémonie, avait été proposée par Duchamp en 1956 comme couverture pour un livre de Rudi Blesh, intitulé Modern Art U.S.A. Ce catalogue précise, à propos de sa propre couverture, qui reproduit ladite jaquette: «it seems appropriate to adopt Duchamp's unused design to clothe a publication for an exhibition of this own work [...] The image [...] reflects Duchamp's fascination with the relation of outer form to inner content («clothes make the mar|»). (II semble pertinent de reproduire le dessin inutilisé de Duchamp pour en revêtir une publication qui traite de son oeuvre exposée. L'image reflète la fascination de Duchamp pour la relation entre la forme extérieure et le contenu interne («l'habit fait le moine»).]

12. Pie-qui-chante, pie-jacasse ou pie-voleuse, celle qui permet de combiner écriture et parole, appelle la lecture des notes p. 127, 379 (note 34) et 300.

13. Ainsi publiée pour la première fois, en couleur et de face, dans l'iconographie duchampienne.

14. Le livre, ne l'oublions pas, est le résultat d'un travail doctoral. 
15. Lisible en anagramme double dans POLLYPERRUQUE. Les mots couvrant les mots, on serait tenté en l'occurrence d'invoquer «l'art-housse» et par là: Larousse, justement impliqué par la planche ornithologique (voir note 30 ).

16. Je souligne: «Enameled», c'est-à-dire littéralement "émaillé». Pour les nombreuses autres relations qui existent entre ces deux oeuvres, voir bien sûr le corps du texte.

17. En banlieue parisienne.

18. Der Witz signifiant le trait d'esprit (comme «wit" en anglais), le jeu de mots, comme chacun sait depuis Freud.

19. La citation in extenso est la suivante: «En appliquant les principes, qu'on ne craigne pas les brèches. II est toujours utile de se souvenir que si les bonnes raisons n'ont pas manqué pour ériger ces principes, cela veut dire seulement que les bonnes raisons ont prévalu sur les raisons opposées. Par ces brèches, on met à jour les raisons opposées.»

20. «ll s'agit, de toute façon, pour dévoyer deux titres posthumes de Raymond Roussel, de domestiquer du même coup "la peau de raie" et "la frange d'or de la petite Paulette"» (p. 119)

21. Pour illustration par l'exemple, ce ready-made, intíulé L.H.O.O.Q. (1919).

22. Numéro de mai 1981, intitulé L'infratexte, couverture de Jean-Pierre Séguin, U.Q.A.C. Voir la note 3 .

23. Ainsi nommée puisque l'éditeur a repris textuellement la description faite par l'auteur.

24. Lieu d'entrecroisement des rets dits «mad», où l'on retrouve le fou des échecs.

25. Le caractère évolutif des définitions et la classification des objets par Duchamp invitent à se référer au chapitre intitulé «Du raide y m'aide» (p. 77-85).

26. Voir p. 119, 120, 123, 124, 127, 154, 371, 375, 378, 389.

27. Voir p. 376-378.

28. Voir la lettre d'Arne Ekstrom à ce sujet, p. 374 (note 20).

29. Très réalistement expédiée de Cadaquès, ibid.

30. Illustration tirée du Larousse pour tous, tome II (1910), ou, plus vraisemblablement, d'une édition ultérieure intitulée Larousse universel (1922-1923, réimprimée jusqu'en 1949). L'auteur de cette planche intitulée «Oiseaux» est Adolphe Millot, «artiste-dessinateur» (1857-1921), d'après une note d'une conférence prononcée par l'auteur en octobre 1987 à Halifax.

31. Qui a donné naissance au psittacisme: «répétition mécanique de phrases, de notions que le sujet n'a ni comprises ni pensées", qui constitue une véritable mise en question de toute forme d'art fondée sur la simple copie conforme.

32. Dans «Zone», ouverture d'Alcools, cité p. 121. Dans un tout autre registre, musical celui-là, on pense à L'oiseau de feu de Manuel de Falla, contemporain de Duchamp et dont l'origine espagnole coïncide avec la référentialité de la carte.

33. Ainsi que l'indique la note 32 , p. 378.

34. Seraient-elles «de Parme" qu'elles appelleraient encore la duplicité, via La chartreuse et la pseudonymie stendhalienne.

35. Oserai-je le qualifier, compte tenu du contexte, d'Andalou (Luis Bunuel)?

36. Avançons celui de Rrose Sélavy. 